\title{
WestVirginiaUniversity
}

THE RESEARCH REPOSITORY @ WVU

Graduate Theses, Dissertations, and Problem Reports

2005

\section{Effects of differential consequences on responding in the analog functional analysis}

Theresa M. V. Hedrick

West Virginia University

Follow this and additional works at: https://researchrepository.wvu.edu/etd

\section{Recommended Citation}

Hedrick, Theresa M. V., "Effects of differential consequences on responding in the analog functional analysis" (2005). Graduate Theses, Dissertations, and Problem Reports. 2209.

https://researchrepository.wvu.edu/etd/2209

This Thesis is protected by copyright and/or related rights. It has been brought to you by the The Research Repository @ WVU with permission from the rights-holder(s). You are free to use this Thesis in any way that is permitted by the copyright and related rights legislation that applies to your use. For other uses you must obtain permission from the rights-holder(s) directly, unless additional rights are indicated by a Creative Commons license in the record and/ or on the work itself. This Thesis has been accepted for inclusion in WVU Graduate Theses, Dissertations, and Problem Reports collection by an authorized administrator of The Research Repository @ WVU. For more information, please contact researchrepository@mail.wvu.edu. 
Effects of Differential Consequences on Responding in the Analog Functional Analysis

Theresa M. V. Hedrick

Thesis submitted to the Eberly College of Arts and Sciences at West Virginia University in partial fulfillment of the requirements for the degree of

Master of Science

In

Psychology

Cynthia M. Anderson, Ph.D. Chair

Katherine Karraker, Ph. D.

Michael Perone, Ph. D.

Department of Psychology

Morgantown, WV

2005

Keywords: Functional Assessment, Developmental Disabilities, Behavior Problems 


\begin{abstract}
Effects of Differential Consequences on Responding in the Analog Functional Analysis
\end{abstract}

Theresa M. V. Hedrick

Direct methods of functional assessment consist of descriptive observations and experimental assessments. Descriptive observations are conducted in non-laboratory settings and consist of recording instances of target responses and environmental events that precede or follow occurrences of problem behavior, whereas experimental assessments most often are conducted in laboratory settings and involve manipulation of only a few predetermined environmental events. Results of recent studies suggest that discrepant hypotheses often are developed based on outcomes of different methods of functional assessment. As intervention efficacy depends on the accuracy of the functional assessment, it is important to determine why discrepant hypotheses often result from descriptive assessments and experimental assessments. One reason may be that a wider array of environmental events occur in descriptive assessments whereas only a few occur in experimental assessments. The purpose of this study was to evaluate whether including different consequences in experimental assessments enhances agreement with descriptive assessments. Two children with developmental disabilities participated. Results were inconclusive due to the small number of participants, variability in results, and lack of intervention data. 


\section{Table of Contents}

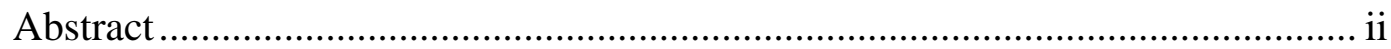

Table of Contents .................................................................................... iii

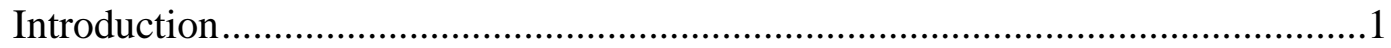

Statement of the Problem...............................................................................6

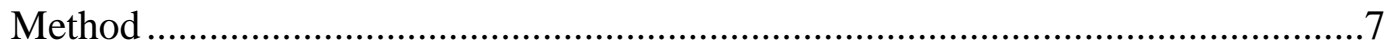

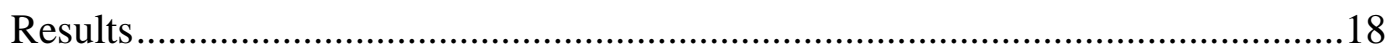

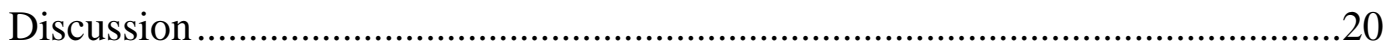

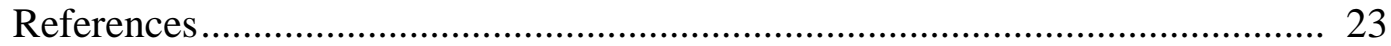


Effects of Differential Consequences on Responding in the Analog Functional Analysis

Treatment of problem behavior exhibited by individuals with disabilities was revolutionized by the development of functional assessment strategies in the 1970's and early 1980’s (Carr et al., 1999). Before this time, interventions often focused on the topography of the problem behavior rather than on environmental variables that affected the occurrence of the response. As a result, interventions focusing on environmental manipulation had to compete with ongoing schedules of reinforcement and thus often consisted of dense schedules of reinforcement using arbitrary reinforcers (stimuli other than those maintaining problem behavior) or punishment. Because methods of functional assessment are useful for developing hypotheses about the relation between environmental events and problem behavior, the development and proliferation of functional assessment strategies resulted in a reduction in the use of contingency management alone (and specifically in the use of punishment) and increases in idiographic interventions (Pelios, Morren, Tesch, \& Axelroad, 1999). A functionally-derived intervention involves manipulating environmental events suggested by a functional assessment to be related to the occurrence of problem behavior. For example, events that evoke problem behavior might be altered, replacement behaviors (responses in the same response class as problem behavior) might be taught and differentially reinforced, the environment might be arranged such that desired responses are more likely to occur, and reinforcing consequences for problem behavior might be removed.

A plethora of methods of functional assessment have been developed in recent years, including both direct and indirect methods. Indirect assessments, include both interviews (e.g., the Functional Analysis Interview; O’Neill, Horner, Albin, Storey, \& Sprague, 1989) and rating scales (e.g., Motivation Assessment Scale, Durand \& Crimmins, 1988) and involve gathering 
information via an informant. In contrast, direct methods of functional assessment are conducted by recording instances of the target response and environmental events as they actually occur. Although indirect methods take less time to conduct, studies to date have not provided strong support for the reliability or validity of indirect methods (e.g., Ellingson, Miltenberger, Stricker, Galensky, \& Garlinghouse, 2000; Shriver, Anderson, \& Proctor, 2001; Sigafoos, Kerr, \& Roberts, 1994; Zarcone, Rodgers, Iwata, Rourke, \& Dorsey, 1991). As a result, the state of the art in functional assessment is to include some type of direct functional assessment in a comprehensive functional assessment (Carr et al., 1999; Horner \& Carr, 1997)

Several methods of direct functional assessment exist including ABC observations (e. g. Bijou, Peterson, \& Ault, 1968), experimental functional analyses (e.g., the analog functional analysis, Iwata, Dorsey, Slifer, Bauman, \& Richman, 1982/1994), and most recently, the structured descriptive assessment (SDA, Anderson \& Long, 2002). Of descriptive methods, ABC assessments, which consist of recording antecedents and consequences surrounding problem behavior, require the researcher to exert the least control over environmental events. These assessments most often are conducted in the setting in which the targeted response typically occurs and involve recording instances of the target response and environmental events that precede and follow the response. The SDA is similar except that specific antecedent conditions demonstrated by previous research to often evoke problem behavior are delivered in a systematic way; consequences are not manipulated. Descriptive assessments such as the ABC functional assessment and the SDA may provide key information about events that are contiguous to problem behavior, however, descriptive assessments do not allow for controlled and systematic manipulation of variables surrounding problem behavior (in the SDA only antecedents are 
manipulated). Thus, causal relations cannot be determined (Iwata, Pace, Kalsher, Cowdery, \& Cataldo, 1990).

Experimental methods of functional assessment (of which the analog functional analysis by Iwata et al., 1982/1994 is most often used in behavior analytic research) allow for the greatest amount of control over environmental variables because predetermined events are manipulated systematically. The analog functional analysis most often is conducted in controlled settings such as laboratories and involves manipulation of specific environmental events using a single-subject experimental design. The analog functional analysis typically consists of five conditions: social disapproval (also referred to as attention), demand, tangible, alone, and control. The purpose of the social disapproval condition is to evaluate whether problem behavior is evoked by attention deprivation and maintained by delivery of attention. The purpose of the demand condition is to evaluate whether problem behavior is evoked by presentations of tasks and maintained by escape or avoidance of task completion. The purpose of the tangible condition is to evaluate whether problem behavior is evoked by the removal of a preferred item and maintained by the return of the item. The purpose of the alone condition is to evaluate problem behavior in the absence of programmed social contingencies. The control condition is used as a comparative condition; no programmed consequences are delivered for the occurrence of problem behavior and attention is delivered on a fixed-time schedule. Hypotheses about environment-behavior relations are developed by comparing relative rates of problem behavior across conditions. For example, if the participant engages in problem behavior primarily in the attention condition, then the results of the analog would suggest that problem behavior was evoked by attention deprivation and maintained by access to attention. 
As discussed above, one important difference between experimental analyses and descriptive assessments is that environmental events are manipulated systematically in experimental analyses. Further, relations are examined between problem behavior and only a few environmental events; presentation of environmental stimuli is controlled such that effects of extraneous variables that may influence problem behavior are reduced. To summarize, control is exerted by (a) manipulating certain events in a systematic fashion, (b) repeating manipulations until stable responding is obtained, (c) using an experimental design to assess functional control, and (d) conducting the assessment in a controlled environment to limit the influence of extraneous variables. Although this degree of control allows the experimenter to make causal statements about functional relations, one tradeoff may be the external validity of the findingsthe degree to which environment-behavior relations identified in the analog functional analysis occur in non-laboratory settings.

One reason for poor agreement may be that because environmental events are not structured in ABC assessments, problem behavior may not occur and/or environmental events that evoke or maintain problem behavior occur rarely (Lerman \& Iwata, 1993). If this is the case, structuring antecedent variables likely to evoke problem behavior may increase the external validity of ABC assessments. To evaluate this hypothesis, Anderson and Long (2002) developed the SDA and compared results obtained via the SDA to results obtained with the analog functional analysis for four children with developmental delays exhibiting problem behavior. For three of four participants (diagnosed with severe to profound mental retardation), the two assessments suggested similar hypotheses about functional relations. For the fourth participant (diagnosed with autism but not mental retardation), differential hypotheses were identified by the two assessments. For this participant, an intervention based on the SDA was implemented in the 
child's classroom and rates of problem behavior were substantially reduced. Unfortunately, an intervention based on the analog was not attempted so there was no way to determine if an intervention based on it might have been more or less effective.

In a follow-up to this study, English (2004) conducted analog functional analyses and SDAs with four children with developmental delay exhibiting problem behavior. One important difference between this study and the study by Anderson and Long (2002) is that three of four participants in Anderson and Long's study were functioning in the severe to profound range of mental retardation; all participants in the study by English were either only mildly delayed or displayed no cognitive delays. In the study by English, differential patterns of responding were observed for all participants in the analogs and the SDAs. English used a component analysis to evaluate interventions based on the analog functional analysis and SDA for all participants. Interventions were conducted by caregivers and tested in the natural environment. For all participants, interventions based on the SDA were more effective than interventions based on the analog functional analysis. Different event-behavior relations have been observed in this study because only specific consequences are manipulated in the analog functional analysis_-results of the SDA suggested that the analog functional analysis did not manipulate the full range of maintaining consequences. For example, in the demand condition of the analog, the only consequence that follows problem behavior is temporary removal of requests. In the SDA, consequences are not programmed and a typical consequence in demand sessions was attention delivery (either alone or in addition to task avoidance), this was determined based on results of conditional probabilities surrounding problem behavior.

Existing research on the SDA (Anderson \& Long, 2002; Anderson, English, \& Hedrick, 2004; English, 2004) and other descriptive assessments (e.g., Lewis, \& Sugai, 1996; Northup et 
al., 1995; Thompson \& Iwata, 2001) suggests that different consequences from those programmed in the analog often maintain problem behavior. For example, Thompson and Iwata conducted ABC observations with 27 adults diagnosed with mental retardation who exhibited problem behavior. Although 21 of the 27 participants engaged in problem behavior following requests, problem behavior was followed by escape for only $36 \%$ of participants and, with the exception of 1 participant (for whom escape almost always occurred), escape was delivered only rarely. Further, tangible delivery almost never followed the occurrence of problem behavior. Recent reviews of the literature on functional analysis suggest that the treatment utility of the analog functional analysis may be limited by the restricted range of consequences assessed and suggest that research is needed evaluating whether additional or alternative consequences (potential reinforcers) should be included in analog functional analyses (Hanley, Iwata, \& McCord, 2003; Sasso et al., 1992).

\section{Statement of the Problem}

Taken together, research suggests that environment-behavior relations identified in the analog functional analysis may differ from those occurring in the natural environment. Further, recent work by English (2004) suggests that interventions based on the SDA may be more efficacious than interventions linked to hypotheses derived from the analog functional analysis. Results obtained by English are compelling as others (e.g., Thompson \& Iwata, 2001) have suggested that descriptive assessments may be less useful than the analog functional analysis for identifying maintaining reinforcers. More research is needed to evaluate whether descriptive assessments such as the SDA have better treatment utility (at least in some situations) than the analog functional analysis. An important step in making this determination involves identifying why results of descriptive and experimental analyses differ. As suggested here, discrepancies 
may occur because consequences identified in descriptive assessments are not tested in the analog.

The purpose of this study was to evaluate effects of including alternative consequences in the analog functional analysis. Two children participated. First, the SDA and analog functional analysis were conducted. Next, for those participants whose SDA suggested that (a) problem behavior in the demand condition may be maintained by attention (instead of or in addition to escape) and/or (b) problem behavior in the tangible condition may be maintained by attention (instead of or in addition to tangible access) contingency manipulations were conducted. In one condition (modified demand), attention was delivered throughout the escape interval. In the other (modified tangible), attention was delivered instead of tangible delivery.

Method

Phase 1: Comparison of Results obtained from the SDA and the Analog Functional Analysis

\section{Participants and Setting}

Two children with developmental delays referred for assessment and treatment of problem behavior participated in this study. Abby was a 4-year-old female diagnosed with autism. She exhibited self-injurious behavior consisting of head banging and face slapping, aggression consisting of biting, pinching, hitting, and kicking, and disruption, consisting of throwing objects, kicking objects, and climbing on furniture. Abby had no functional speech but could follow simple directions. Matt was a 4-year-old male diagnosed with pervasive developmental disorder not otherwise specified. He exhibited self-injurious behavior (SIB) consisting of throwing himself to the floor, aggression consisting of hitting, kicking, and choking others, and disruption consisting of throwing objects, kicking objects, spitting, and blowing mucus. Matt had some verbal communication and could follow simple instructions. The SDA 
was conducted in participants' homes. The analog functional analysis was conducted in the Applied Behavior Analysis laboratory at West Virginia University. Sessions for both the SDA and the analog functional analysis lasted for 10 minutes and were conducted 3 to 5 times a week for 1 to 2 hour periods.

Response Definitions, Data Collection, and Interobserver Agreement

Structured descriptive assessment. Data were collected using a real time computerized observing system. All sessions were videotaped for later scoring. Data were collected for child and caregiver responses. Child responses included problem behavior (individually defined and coded as a frequency measure) and compliance, coded as a partial interval measure across consecutive 5-s intervals and defined as completing a request within one 5-s interval of a prompt.

Caregiver responses were coded as partial interval measures across consecutive 5-s intervals. The following responses were coded: prompts, escape, attention deprivation, attention delivery, tangible removed or denied, and tangible delivery. Prompts were defined as an instruction to complete a previously identified task (prior to conducting the assessments, caregivers were asked to list tasks they frequently asked the child to complete). Prompts included verbal instructions, physical prompts, and the presence of an ongoing physical context (e.g., seated at a table with a workbook). Prompts for Abby consisted of picking up her toys and handing items to her mother or father. Prompts for Matt consisted of picking up his toys and placing them in a toy box and sitting at the table. Escape was coded if, for an entire 5-s interval following the delivery of a prompt, the participant was not engaged in a predefined task and prompts to engage in the task were not emitted. Attention deprivation was coded after 1 complete interval elapsed during which no attention or prompts occurred. Attention delivery was coded when the caregiver interacted with the child in a non-instructional manner. This included neutral 
statements, verbal reprimands, and physical attention (e.g. a hug or restraint). Tangible removed or absent was coded when the caregiver removed a predefined preferred item from the child. Tangible delivery was coded when the caregiver delivered or allowed the child to access the predefined preferred item. Abby’s preferred item was the television and a stuffed bear (a Carebear); for Matt the preferred item was the television.

Prior to beginning data collection, coders were trained on the data collection system using video recordings obtained from previous studies. Training continued until interobserver agreement coefficients of $80 \%$ or greater were achieved on all target responses across three consecutive sessions. Once data collection has commenced, if interobserver agreement had fallen below $80 \%$ on any code for three consecutive sessions, coders would have been retrained following the original protocol; this did not occur during the study. Interobserver agreement was collected on 33\% of all sessions. Agreement coefficients for partial interval data were calculated based on occurrence, nonoccurrence, and total agreement. Occurrence agreement was calculated by dividing the number of intervals both observers coded a response by the total number of intervals either observer coded a response and multiplying the resulting proportion by 100 . Nonoccurrence agreement was calculated by dividing the number of intervals both observers did not code a response by the number of intervals either observer did not code a response and multiplying the resulting coefficient by 100 . Total agreement was calculated by dividing the number of intervals in which both observers agreed on occurrence or nonoccurrence of a response by the total number of intervals, and multiplying by 100. Agreement coefficients for frequency data were calculated by dividing sessions into 10-s intervals and within each interval, dividing the smaller number of responses coded by one observer by the larger number of responses coded by the second observer. For each session coded for reliability, one coded served 
as the primary coder. In the case of disagreement, the primary coders codes were used for data analysis. These totals were then summed and divided by 60 (the total number of 10-s intervals), then multiplied by 100 to obtain an agreement coefficient.

Analog functional analysis. Data were collected using a real time computer based observing system. The sessions were videotaped for later scoring. Child and therapist responses were scored as frequency measures. Child problem behavior was defined as per the SDA but compliance was defined as completing a requested task following a verbal or gestural prompt. Therapist responses included three types of prompts (verbal, gestural, physical), attention delivery, tangible delivery, and tangible removed or denied. All therapist responses were scored as frequency measures. In the analog functional analysis, tasks used in the demand condition and tangible items used in the tangible condition were identical to those used in the SDA.

Verbal prompts were coded when the therapist delivered a verbal request to the child. Gestural prompts were coded when the therapist repeated the verbal prompt and simultaneously modeled the correct response. Physical prompts were coded when the therapist physically guided the child to complete the task. Attention delivery was defined as brief verbal comments or physical attention that is not a prompt, including reprimands (e.g., “don’t hit me,”) and neutral statements (e.g., “you are playing nicely.”). Tangible delivery was coded if the therapist allowed the child access to a preferred item (identical tangible items were used in both the SDA and the analog). Tangible removal was coded if the therapist removed a preferred item from the child. Escape was coded if, in the absence of compliance, the therapist removed the task materials from the child and did not continue to deliver prompts to the child for 5 consecutive seconds.

Data collection, observer training, and calculation of interobserver agreement was the same as for the SDA. 


\section{Integrity Measurement}

To develop hypotheses about functional relations in the SDA, antecedent conditions must be delivered in a structured and systematic way. Following the conclusion of the SDA, the proportion of intervals in which antecedents were present in each condition of the SDA was calculated to ensure that relevant antecedent conditions occurred in various conditions of the SDA (e.g., prompting occurred during the task condition but not during the attention condition). Intervals scored with relevant antecedents are depicted in Table 1. For example, for Matt 96.4\% of the intervals in the attention condition sessions were scored with attention deprivation and $0 \%$ of the intervals in the attention condition sessions were scored with task presentation. Experimental Design and Procedure

A caregiver interview was conducted prior to the start of assessments. The interview was used to identify prompts that were used in the demand conditions of both assessments and preferred items that were used in tangible conditions of the two assessments.

Structured descriptive assessment. The SDA was conducted as described by Anderson and Long (2002). The SDA was conducted in a multielement design. Participants were exposed repeatedly to each of four conditions, demand, tangible, attention, and play, in random order, except no more than two consecutive presentations of a condition. The caregiver was instructed to respond to problem behavior as he or she normally would. Four sessions per condition were conducted. Visual inspection was used as the basis for stability. To the extent possible, conditions were conducted during times the antecedent likely would naturally occur.

The purpose of the demand condition was to establish the antecedent of task presentation. The caregiver was instructed to present the child with tasks from the previously established list. 
If in the absence of ongoing compliance or problem behavior, the caregiver failed to deliver prompts for one consecutive minute, the caregiver was instructed to resume prompting.

The purpose of the tangible condition was to establish the antecedent of tangible removal. The caregiver was instructed to allow the child at least 2 min access to the item prior to the start of the condition. The caregiver was then instructed to remove the preferred item. If the child was allowed access to the item for $1 \mathrm{~min}$ in the absence of problem behavior, the caregiver was instructed to remove the item.

The purpose of the attention condition was to establish the antecedent of attention deprivation. Prior to the start of the condition, the caregiver was instructed to spend 2 min directly interacting with the child in a non-instructional manner. Predefined preferred tangible items were not present during this time or during the attention condition. When the session began, the parent was instructed to engage in an activity that did not allow for him or her to directly interact with the child (e.g. talking on the telephone or preparing a meal). In the absence of problem behavior, if the caregiver interacted with the child for longer than $1 \mathrm{~min}$, the caregiver was instructed to return to an activity that did not allow him or her to directly interact with the child.

The play condition was designed to be somewhat analogous to the play condition of the analog functional analysis. The child had access to preferred items, was not presented tasks, and caregiver attention was delivered. The caregiver was instructed to play with the child and to refrain from presenting demands to the child. If the caregiver failed to deliver attention for longer than 1 min, the caregiver was instructed to interact with the child.

Analog functional analysis. The analog functional analysis was conducted in a similar manner to that described by Iwata et al. (1984/1992). During the analog functional analysis 
participants were repeatedly exposed to four conditions: demand, tangible, attention, and play. Conditions were presented in random order, except that no more than two consecutive presentations of a condition occurred. A minimum of four sessions per each condition were conducted and continued until stability, judged by visual inspection, was reached.

For Abby, trained research assistants from the West Virginia University Department of Psychology served as therapists during the analog functional analysis. The analog functional analysis with Matt was initially conducted by trained therapists, however, Matt did not exhibit any problem behavior for 8 consecutive sessions across conditions, and thus his mother conducted the functional analysis.

The purpose of the demand condition was to evaluate whether task presentation evoked problem behavior and escape maintained problem behavior. During this condition tasks were presented in a three-step hierarchy consisting of sequential verbal, gestural, and physical prompts. Tasks were similar to those used in the SDA. A brief positive statement (e.g. "Nice working”) was delivered to the participant contingent on compliance. If the participant engaged in problem behavior, all prompts and materials were removed for 20 s. During the 20-s escape period no programmed consequences were delivered following problem behavior.

The purpose of the tangible condition was to evaluate whether tangible removal evoked problem behavior and tangible delivery maintained problem behavior. Preferred items were the same as those used in the SDA. Prior to the start of the tangible condition participants were allotted a 2-min period of free access to preferred items. The preferred items were removed from the participant at the start of the session. Preferred items were returned for 20-s following any instance of problem behavior. There were no programmed consequences for problem behavior occurring during the 20-s access to the preferred item. 
The purpose of the attention condition was to evaluate whether attention deprivation evoked problem behavior and attention delivery maintained problem behavior. During the attention condition the participant had access to low preference tangible items. The therapist engaged in an activity that does not allow direct interaction with the child (e.g. pretending to read a book or magazine) and delivered a brief social-negative statement (e.g. "I don't like that,” “It is not nice to hit," “That hurts”) on a FR 1 schedule contingent upon the occurrence of problem behavior.

The purpose of the play condition was to control for the presence of the therapist, the presence of preferred items, and the absence of demands. During the play condition the therapist delivered social-positive attention (e.g. "I like the way you are playing”) on a FT 20-s schedule. If problem behavior occurred within $5 \mathrm{~s}$ of the scheduled delivery of attention, attention was withheld until 5 s had elapsed during which no problem behavior occurred. There were no programmed consequences for problem behavior.

\section{Data Analysis}

For the SDA, conditional probabilities were calculated to assess the relation between antecedent and consequent events and problem behavior. Conditional probabilities were calculated based upon the procedures described by English (2004). Proportions that were calculated are depicted in Table 1. Two proportions were calculated for each consequent event, a behavior-based proportion and an event-based proportion. The numerator for each proportion is the same however the denominator differs; the denominator for the behavior-based proportion is intervals scored with problem behavior, the denominator for the event-based proportion is intervals scored with the event. 
Behavior-based probabilities reveal the proportion of intervals scored with problem behavior that were followed by a specific event within 5-s. Thus, this proportion reveals the putative schedule of reinforcement; the closer the resulting proportion is to 1 , the richer the schedule. Event-based proportions were used to determine the proportion of intervals scored with a specific event that followed a target response (e.g., the proportion of intervals scored with attention that followed problem behavior). The event-based proportion provided information about dependency — the closer the resulting proportion is to 1, the stronger the dependency. For example, if the behavior-based proportion for attention delivery was .85 and the event-based proportion was .91, then $85 \%$ of intervals scored with problem behavior were followed by attention and $91 \%$ of all attention deliveries that were scored followed problem behavior.

All probabilities were coded based on the first occurrence of child behavior in each interval (as if child behavior was coded using a partial-interval procedure) and proportions were calculated only for intervals in which relevant antecedents were in place. Thus, escape as a consequence was calculated only in the presence of prompts (which might establish escape as reinforcing), attention delivery and tangible delivery as consequences was evaluated in the presence of attention deprivation, prompts, and tangible denial (all of which might establish these consequences as reinforcing).

\section{Phase 2: Manipulation of Consequences}

\section{Participants and Setting}

Participants were the same as in experiment 1 . Sessions were conducted in an observation room located in the Department of Psychology at West Virginia University or in the participant's home.

Response Definitions, Data Collection, Interobserver Agreement, and Integrity Measures 
Response definitions, data collection procedures, interobserver agreement are the same as in the analog functional analysis in experiment 1 . Integrity measures were the same as in the analog functional analysis in Phase 1.

Experimental Design and Procedure.

Demand consequence manipulation. The purpose of conducting manipulations of consequences in the demand condition was to evaluate whether the occurrence of problem behavior was differentially affected by delivery of attention in the escape interval. The demand consequence manipulation consisted of two conditions: demand/escape and demand/escape plus attention. These conditions were presented in a multielement design in random order with no more than two consecutive presentations of one condition. To facilitate discrimination between conditions different environmental stimuli (e.g., different rooms and/or different clothing) were used for the demand/escape condition and the demand/escape attention condition. Three sessions per condition were conducted. The demand consequence manipulations conditions continued until stability was reached or a maximum of 8 sessions per condition were conducted. Stability was based on visual inspection.

The demand/escape condition was conducted in the same manner as the demand condition during the analog functional analysis. During the demand/escape plus attention condition, prompts were delivered and consequences for compliance were the same as in the analog functional analysis. If the participant engaged in problem behavior, all prompts and materials were removed for 20 seconds. During the 20-s escape period social-negative attention was delivered on a continuous schedule (i.e. the therapist discussed why it is wrong to hit or throw materials). 
Tangible consequence manipulation. The purpose of the tangible consequence manipulation was to evaluate whether the occurrence of problem behavior was differentially affected by attention delivery when the tangible item is removed. The tangible consequence manipulations consisted of two conditions: tangible/tangible and tangible/attention condition. The experimental design, methods to enhance discrimination, and stability criterion were identical to the demand consequence manipulation. The tangible/tangible condition was conducted exactly the same as the tangible condition during the analog functional analysis. During the tangible/attention condition the preferred item was removed at the start of the condition. Instances of problem behavior during this condition were followed by social-negative attention similar to that delivered in the attention condition of the analog functional analysis. During the tangible/attention condition the therapist did not return the preferred item during the session.

\section{Data Analysis}

Demand consequence manipulations. To determine results of the tangible consequence manipulation, data were evaluated through visual inspection of line graphs. In addition, the proportion of responses occurring during the escape interval was calculated and compared across the two conditions.

Tangible consequence manipulations. To determine results of the demand consequence manipulation, data was evaluated through visual inspection of line graphs. In a subsequent analysis, problem behaviors per minute occurring during the tangible absent portions of the tangible/tangible condition were compared to mean responses occurring during the tangible/attention condition (to control for the presence of the preferred item). 


\section{Results}

For each participant, results of the SDA, the analog functional analysis, and the contingency manipulations are depicted in Figures 1 through 4. For ease of interpretation, conditional probabilities for the SDA are depicted only for those conditions in which relevant antecedents were in effect for a significant portion of the session. Thus, proportions in the presence of attention deprivation are depicted if attention deprivation was present for an average of $65.2 \%$ of the time during sessions of a given condition for Abby and $96.4 \%$ of the time during sessions of a given condition for Matt. Proportions in the presence of tangible denied are depicted if tangible deprivation was in effect for $70 \%$ or more of a condition, and proportions in the presence of prompts are depicted if prompts were in effect for at least $40 \%$ of sessions in a given condition. In addition, event-based proportions are not graphed, but rather are discussed in the text when relevant.

Abby

Results obtained with Abby are depicted in Figures 1and 2. Results from the analog functional analysis (top panel) suggest that problem behavior was multiply maintained. Problem behavior was evoked by attention deprivation and maintained by attention delivery. In addition, elevated responding in the tangible condition suggested that removal of preferred items evoked problem behavior and that return of those items maintained responding. Finally, prompts evoked problem behavior and, in the presence of prompts, task avoidance maintained responding.

Abby exhibited elevated responding in the attention, tangible, and demand conditions of the SDA as well (middle panel), but, with the exception of the attention condition (during which attention delivery frequently followed problem behavior), conditional probabilities suggest different hypotheses about maintaining consequences relative to results obtained in the analog 
functional analysis. In the demand condition, escape occurred only rarely following problem behavior but almost $63 \%$ of problem behavior was followed by attention, suggesting that attention delivery might maintain responding (in addition to or instead of escape from tasks) in the presence of prompts. Further, in the tangible condition, tangible delivery never followed problem behavior but attention delivery occurred following $89 \%$ of intervals scored with problem behavior, suggesting that attention might maintain responding in the presence of tangible removal. Interestingly, event-based proportions revealed that attention delivery (in all conditions) and escape (in the demand condition) were far more likely to occur independent of problem behavior. For example, in the demand condition (and in the presence of prompts), only $18 \%$ of all attention deliveries and $17 \%$ of all escape deliveries followed problem behavior. In the attention condition, in the presence of attention deprivation, only 19\% of all the attention delivered followed problem behavior.

The results of the consequence manipulations are depicted in Figure 2. The tangible manipulations are depicted in the top panel. During these manipulations, problem behavior occurred at higher rates during the condition in which problem behavior was followed by access to the preferred item. Although responding initially was elevated in the attention delivery condition, after the first session, responding occurred only rarely in this condition. The demand consequence manipulations are depicted in the bottom panel of Figure 2. Abby exhibited higher rates of problem behavior when attention was delivered during the escape interval then when no attention occurred during the escape interval. During these manipulations, Abby exhibited $81.0 \%$ of problem behavior during prompting and $19.0 \%$ of problem behavior during the escape interval of the attention during escape condition, and $88.9 \%$ of problem behavior during prompting and $19 \%$ of problem behavior during the escape interval of the no attention during escape condition. 
Matt

Results obtained with Matt are depicted in Figures 3 and 4. As is shown in the top panel of Figure 3, Matt exhibited elevated rates of responding in all conditions except the play condition, suggesting that, as was true with Abby, problem behavior was multiply maintained. The results of the SDA are depicted in the middle and bottom panels. Matt exhibited elevated responding in the demand condition of the SDA (middle panel), and conditional probabilities reveal that, in the presence of prompts, problem behavior was most often followed by attention, but escape occurred occasionally as well. As was true with Abby, event-based proportions revealed that both escape and attention delivery were more likely to occur independent of responding.

The contingency manipulations were only conducted in the demand consequence manipulations due to the low occurrence of problem behavior in the tangible condition in the SDA. As was the case with Abby, Matt exhibited higher rates of problem behavior when attention was delivered in the escape interval. During these manipulations, Matt exhibited 94.1\% of problem behavior during prompting and 5.9\% of problem behavior during the escape interval of the attention during escape condition, and 100\% of problem behavior during prompting and $0 \%$ of problem behavior during the escape interval of the no attention during escape condition

\section{Discussion}

Previous research using the SDA suggests that problem behavior may be maintained by a broader range of consequences than typically is manipulated in the analog functional analysis. For example, in the presence of prompts, attention delivery (in addition to or instead of escape) may play an important role. The purpose of this study was to evaluate effects of including different consequences in the analog functional analysis. 
Both participants exhibited elevated responding in the demand condition of the SDA and the analog functional analysis, however, whereas the analog suggested that problem behavior was maintained only by escape (as this is the only consequence tested), the SDA suggested that attention delivery might be important as well. When attention delivery occurred during the escape interval of analog sessions, both participants emitted higher levels of responding relative to sessions when escape did not contain attention delivery. One participant exhibited elevated responding in the tangible condition of both assessments but the SDA suggested that problem behavior might be maintained by attention; tangible delivery never occurred following problem behavior in the SDA. In follow-up analyses, attention delivery occurred following problem behavior in the tangible condition of the analog, however, lower rates of responding were observed then when problem behavior was followed by brief access to the preferred item. This finding may have occurred for several reasons. First, it is possible that the results of the SDA were not accurate and that access to tangibles maintained problem behavior but attention delivery did not. Differential results may, however, have been due to who conducted the assessments. The SDA was conducted by the participant's mother whereas the analog and follow-up analyses for Abby were conducted by experimenters. It is possible that for Abby, mother's attention served as a reinforcer whereas attention from experimenters did not.

In sum, the results of this study are inconclusive given the limited number of participants, the variability in results, and the lack of intervention data demonstrating the treatment utility of hypotheses derived from the two assessments. An increase in participants, would allow for evaluation of variability in results to determine if variability in hypothesis suggested by the assessments was an individual case or a trend among participants. Future research should continue to evaluate effects of including a broader range of stimuli in the analog functional 
analysis and especially should evaluate effects of such manipulations on intervention outcomes. Manipulation of intervention outcomes would evaluate the treatment utility of each assessment and allow for evaluation of environment-behavior relations surrounding problem behavior (English, 2004). 


\section{References}

Achenbach, T. M. (1991). Manual for Child Behavior Checklist 4-18 1991 Profile. Burlington, VT: University of Vermont Department of Psychiatry.

Anderson, C. M., English, C., \& Hedrick, T. (in press). Use of the structured descriptive assessment with typically developing children. Behavior Modification.

Anderson, C. M., Freeman, K. A., \& Scotti, J. R. (1999). Evaluation of the generalizability (reliability and validity) of analog functional assessment methodology. Behavior Therapy, 30, 31-50.

Anderson, C. M., \& Long, E. S. (2002). Use of a structured descriptive assessment methodology to identify variables affecting problem behavior. Journal of Applied Behavior Analysis, 35, 137-154.

Bijou, S. W., Peterson, R. E., \& Ault, M. H. (1968). A method to integrate descriptive and experimental field studies at the level of data and empirical concepts. Journal of Applied Behavior Analysis, 1, 175-191.

Carr, E. G., Horner, R. H., Turnbull, A. P., Marquis, J. G., McLaughlin, D. M., McAtee, M. L., et al. (1999). Positive behavior support for people with developmental disabilities: A research synthesis. Washington, DC: American Association on Mental Retardation.

Conroy, M. A., Fox, J., Crain, L., Jenkins, A., \& Belcher, K. (1996). Evaluating the social and ecological validity of analog assessment procedures for challenging behaviors in young children. Education and Treatment of Children, 19, 233-256.

Conroy, M. A., \& Stichter, J. P. (2003). The application of antecedents in the functional assessment process. Journal of Special Education, 37, 15-26. 
Durand, V. M., \& Crimmins, D. B. (1988). Identifying the variables maintaining self-injurious behavior. Journal of Autism and Developmental Disorders, 18, 99-117.

Ellingson, S. A., Miltenberger, R. G., Stricker, J., Galensky, T. L., \& Garlinghouse, M. (2000). Functional assessment and intervention for challenging behaviors in the classroom by general classroom teachers. Journal of Positive Behavior Interventions, 2, 85-97.

English, C. L., \& Anderson, C. M. (in press). Evaluation of the treatment utility of the analog functional analysis and the structured descriptive assessment. Journal of Positive Behavior Interventions.

English, C. L., \& Anderson, C. M. (2004). Effects of familiar therapists on responding in the analog functional analysis. Research in Developmental Disabilities, 15, 39-55.

Hanley, G. P., Iwata, B. A., \& McCord, B. E. (2003). Functional analysis of problem behavior: A review. Journal of Applied Behavior Analysis. 36, 147-185

Horner, R. H., \& Carr, E. (1997). Behavioral support for students with severe disabilities: Functional assessment and comprehensive intervention. Journal of Special Education, 34, 84-109.

Horner, R. H., Day, H. M., \& Day, J. R. (1997). Using neutralizing routines to reduce problem behaviors. Journal of Applied Behavior Analysis, 30, 601-614.

Iwata, B. A., Dorsey, M. E., Slifer, K. J., Bauman, K. F., \& Richman, G. S. (1994). Toward a functional analysis of self-injury. Journal of Applied Behavior Analysis, 27, 197-209.

Iwata, B. A., Pace, G. M., Kalsher, M. J., Cowdery, G. E., \& Cataldo, M. F. (1990). Experimental analysis and extinction of self-injurious escape behavior. Journal of Applied Behavior Analysis, 23, 11-27. 
Lerman, D. C., \& Iwata, B. A. (1993). Descriptive and experimental analysis of variables maintaining self-injurious behavior. Journal of Applied Behavior Analysis, 26, 293-319.

Lewis, T. \& Sugai, G. (1996). Descriptive and experimental analysis of teacher and peer attention and the use of assessment-based intervention to improve pro-social behavior. Journal of Behavioral Education, 6, 7-24.

Mueller, M. M., Wilczynski, S. M., Moore, J. W., Fusshier, I., \& Trahant, D. (2001). Antecedent manipulations in a tangible condition: Effects of stimulus presentation on aggression. Journal of Applied Behavior Analysis, 34, 237-240.

Murphy, E. S., McSweeney, F. K., Smith, R. G., \& McComas, J. J. (2003). Dynamic changes in effectiveness: Theoretical methodological and practical implication for applied research. Journal of Applied Behavior Analysis, 36, 421-428.

Northup, J., Broussard, C., Jones, K., George, T., Vollmer, T. R., \& Herring, M. (1995). The differential effects of teacher and peer attention on the disruptive classroom behavior of three children with a diagnosis of attention deficit hyperactivity disorder. Journal of Applied Behavior Analysis, 28, 227-228.

O’Neill, R. E., Horner, R. H., Albin, R. W., Storey, K., \& Sprague, J. R. (1989). The functional analysis interview. In R. H. Horner, J. L. Anderson, E. G. Carr, G. Dunlap, R. L. Koegel, \& W. Sailor (Eds.). Functional analysis: A practical assessment guide (pp. 10-23). Eugene: University of Oregon Press.

Pelios, L., Morren, J., Tesch, D., \& Axelrod, S. (1999). The impact of functional analysis methodology on treatment choice for self-injurious and aggressive behavior. Journal of Applied Behavior Analysis, 32, 185-195 
Sasso, G. M., Reimers, T. M., Cooper, L. J., Wacker, D., Berg, W., Steege, M., et al. (1992). Use of descriptive and experimental analyses to identify the functional properties of aberrant behavior in school settings. Journal of Applied Behavior Analysis, 25, 809-821.

Shriver, M. D., Anderson, C. M., \& Proctor, B. (2001). Evaluating the validity of functional behavior assessment. School Psychology Review, 30, 180-192.

Sigafoos, K, Kerr, M., \& Roberts, D. (1994). Interrater reliability of the Motivation Assessment Scale: Failure to replicate with aggressive behavior. Research in Developmental Disabilities, 15, 333-42.

Thompson, R. H., \& Iwata, B. A. (2001). A descriptive analysis of social consequences following problem behavior. Journal of Applied Behavior Analysis, 34, 169-178.

Van Camp, C. M., Lerman, D. C., Kelley, M. E., Roane. H. S., Contrecci, S. A., \& Vorndran, C. M. (2000). Further analysis of idiosyncratic antecedent influences during the assessment and treatment of problem behavior. Journal of Applied Behavior Analysis, 33, 207-221.

Vollmer, T. R., Borrero, J. C., Wright, C. S., Van Camp, C., \& Lalli, J. S. (2001). Identifying possible contingencies during descriptive analyses of severe behavior disorders. Journal of Applied Behavior Analysis, 34, 269-287.

Zarcone, J. R., Rodgers, T. A., Iwata, B. A., Rourke, D. A., \& Dorsey, M. F. (1991). Reliability analysis of the Motivation Assessment Scale: a failure to replicate. Research in Developmental Disabilities, 12, 349-360. 
Table 1. Percentage of intervals an antecedent was present during relative conditions in the structured descriptive assessment.

\begin{tabular}{|c|c|c|c|c|}
\hline Abby & \multicolumn{4}{|c|}{ Condition } \\
\hline Antecedent & Attention & Demand & Tangible & Play \\
\hline Attention Deprivation & 67.1 & 1.8 & 5.1 & 9.1 \\
\hline Task Presentation & 0 & 79.5 & 0 & 0 \\
\hline Tangible Removed or & 100 & 100 & 84.1 & 0 \\
\hline \multicolumn{5}{|l|}{ Absent } \\
\hline Matt & \multicolumn{4}{|c|}{ Condition } \\
\hline Antecedent & Attention & Demand & Tangible & Play \\
\hline Attention Deprivation & 96.4 & 23.1 & 7.5 & 9.1 \\
\hline Task Presentation & 0 & 72.5 & 0 & 0 \\
\hline Tangible Removed or & 100 & 100 & 0 & 0 \\
\hline Absent & & & & \\
\hline
\end{tabular}


Table 2. Formulas Used to Calculate Conditional Probabilities for the Structured descriptive assessment.

Escape as a consequence

Attention as

a

consequence

Tangible

delivery as a

consequence
Number of intervals with escape deliveries that followed problem behavior Number of intervals with escape deliveries

Number of intervals with escape deliveries that followed problem behavior Number of intervals with problem behavior

Number of intervals with problem behavior followed by attention delivery Total number of intervals with attention deliveries

Number of intervals with problem behavior followed by attention delivery Total number of intervals with problem behaviors

Number of intervals with problem behavior followed by tangible delivery Total number of intervals with tangible deliveries

Number of intervals with problem behavior followed by tangible delivery Total number of intervals with problem behavior 


\section{Figure Caption}

Figure 1. The top graph shows results of the analog functional analysis, the middle graph shows the results of the structured descriptive assessment, and the bottom graph shows the behaviorbased proportions present in the structured descriptive assessment for Abby. Figure 2. The top panel shows the results of the tangible consequence manipulation and the bottom panel shows the results of the tangible consequence manipulation for Abby.

Figure 3. The top graph shows results of the analog functional analysis, the middle graph shows the results of the structured descriptive assessment, and the bottom graph shows the behaviorbased proportions present in the structured descriptive assessment for Matt.

Figure 4. The results of the demand consequence manipulation for Matt. 
Figure 1.

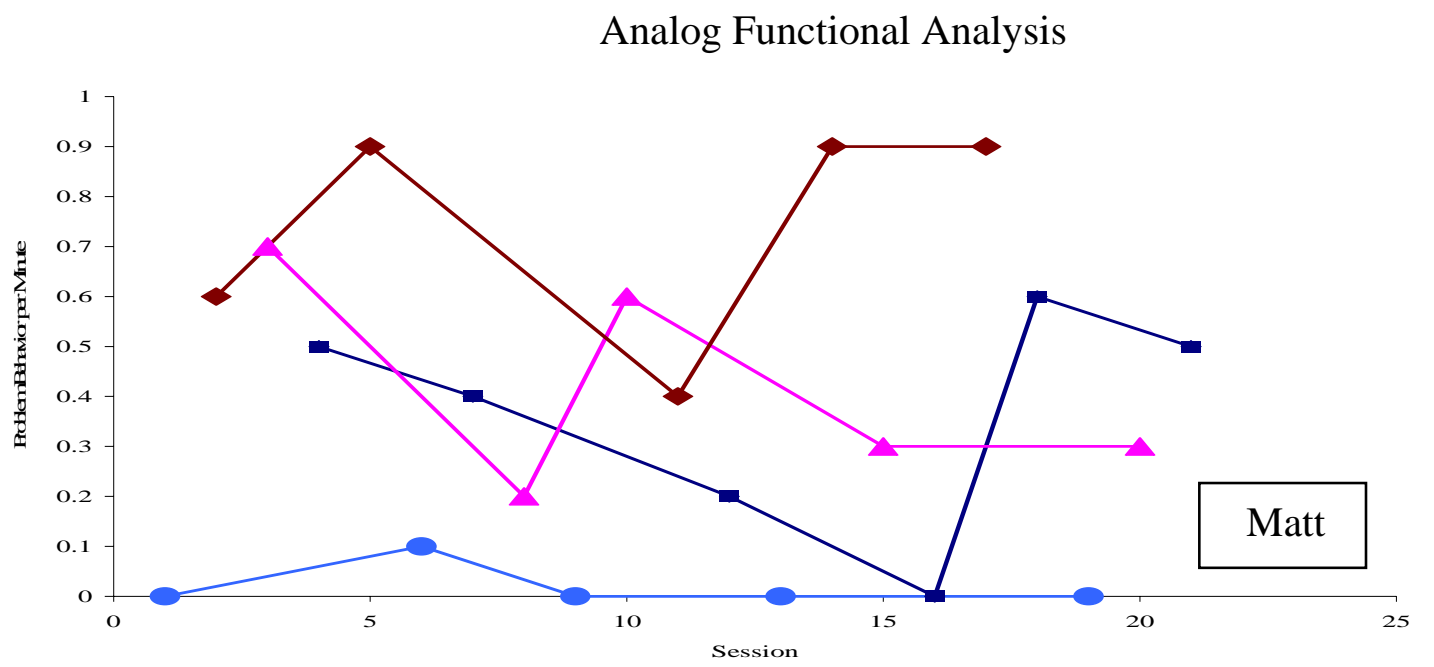

Structured Descriptive Assessment

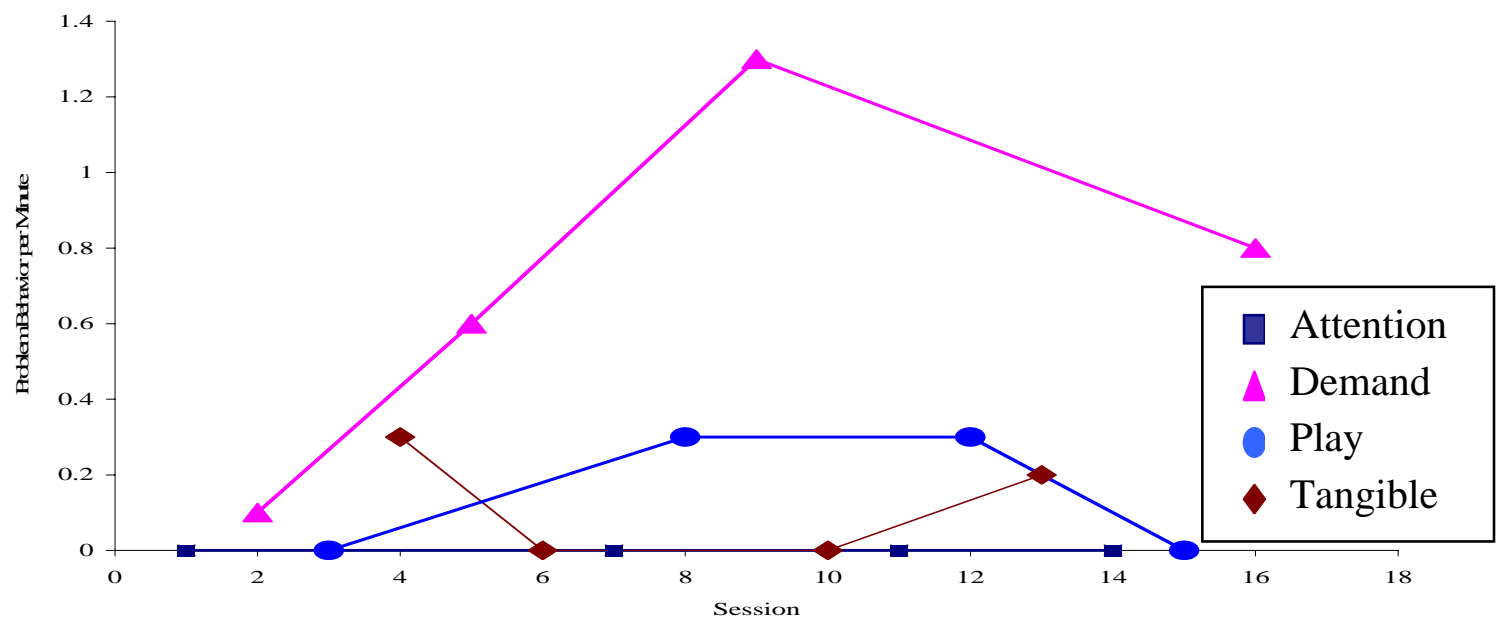

Behavior-Based Probabilities

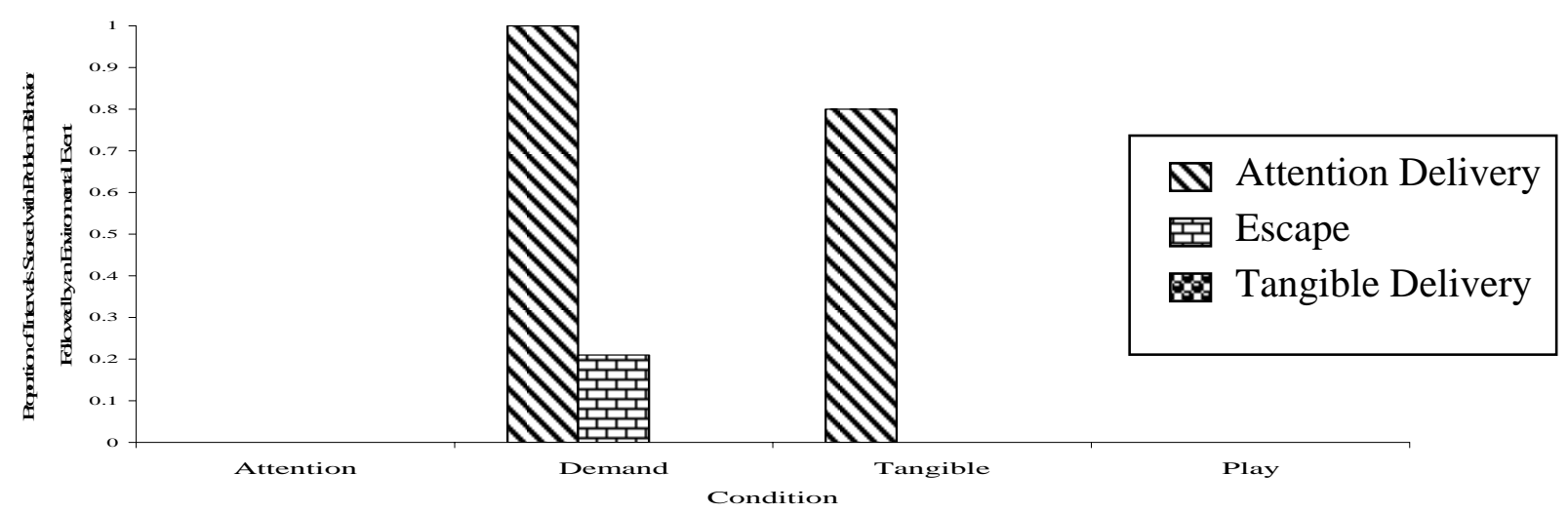


Differential consequences 31

Figure 2.

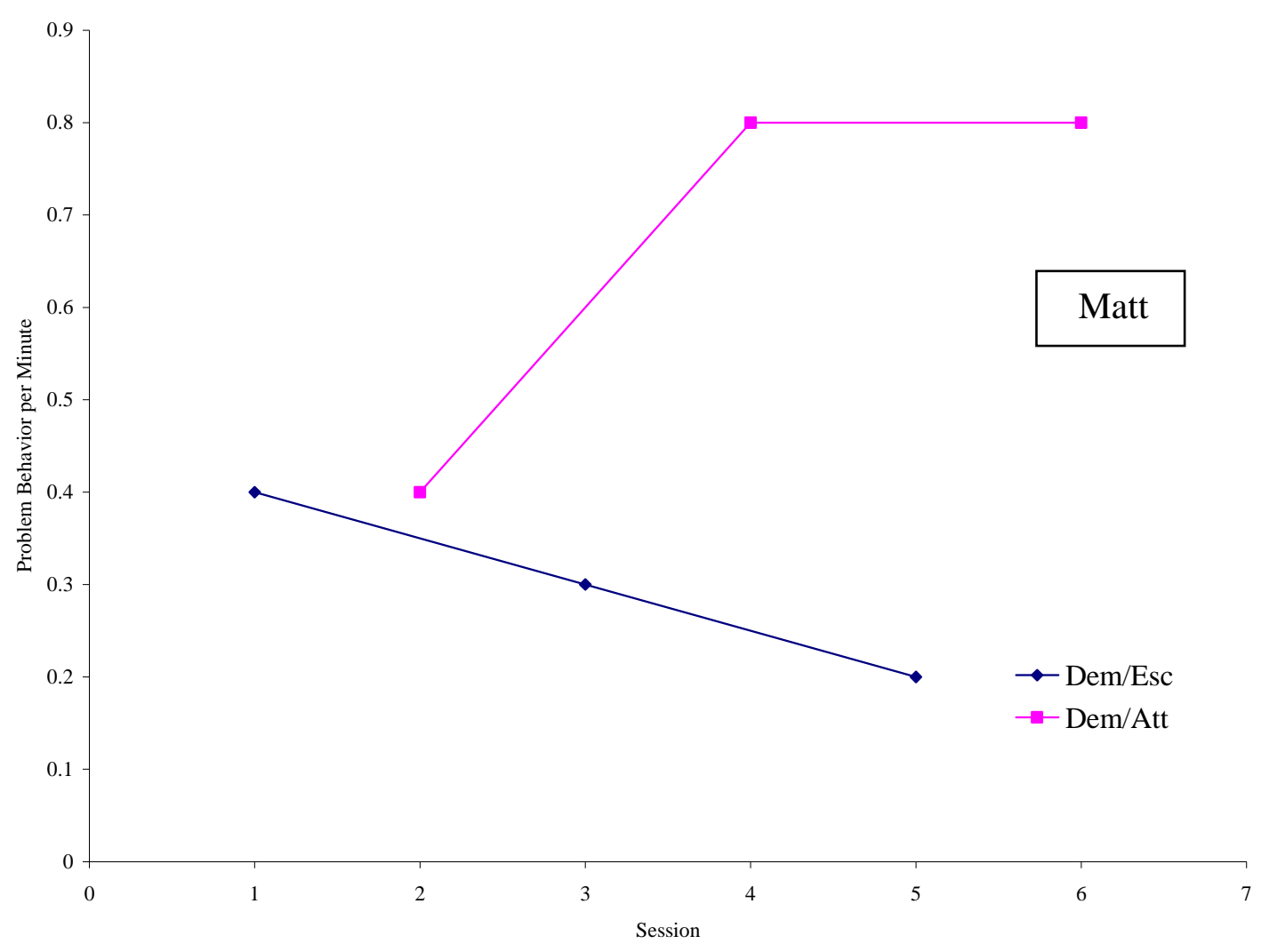


Figure 3.

\section{Analog Functional Analysis}

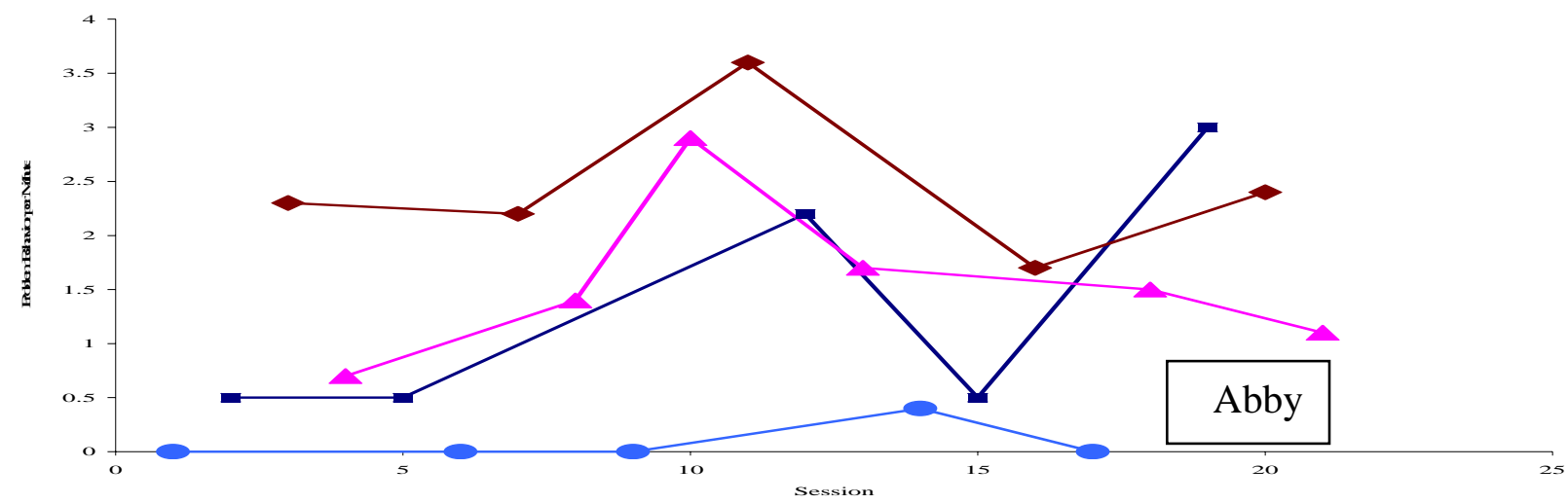

Structured Descriptive Assessment

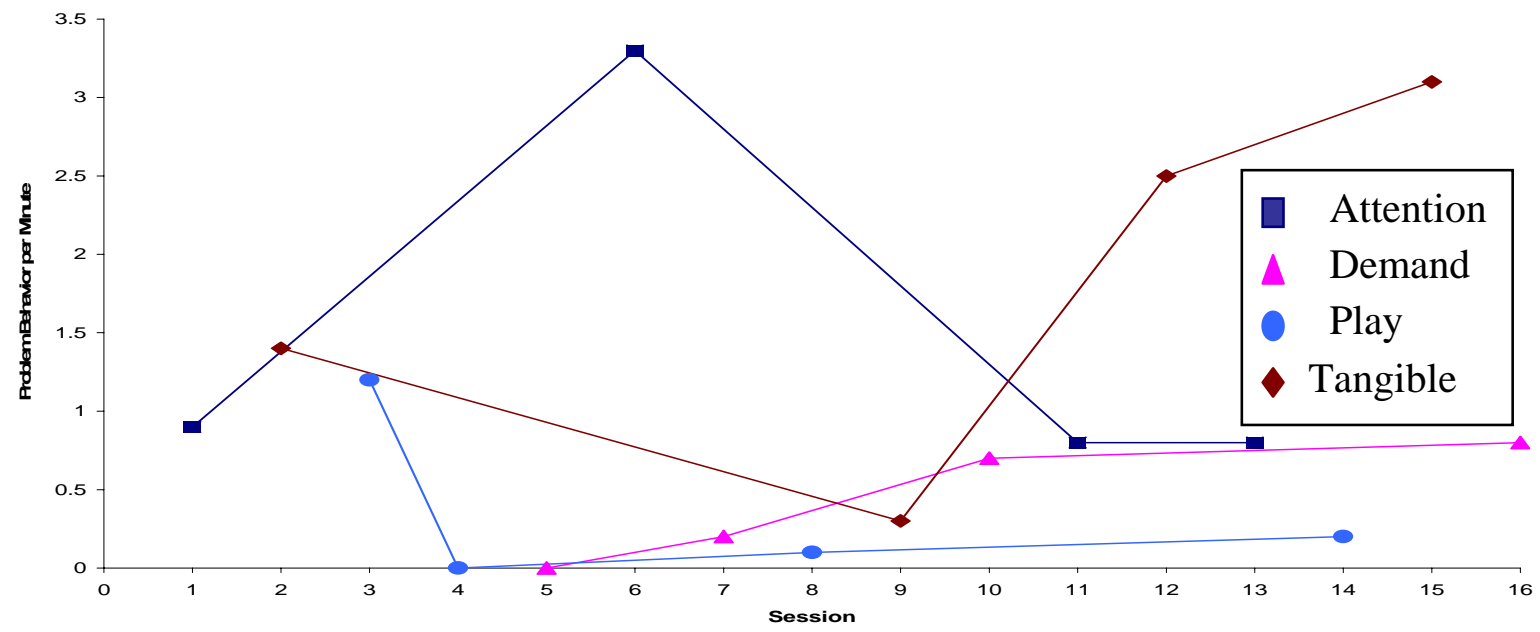

Behavior-Based Probabilities

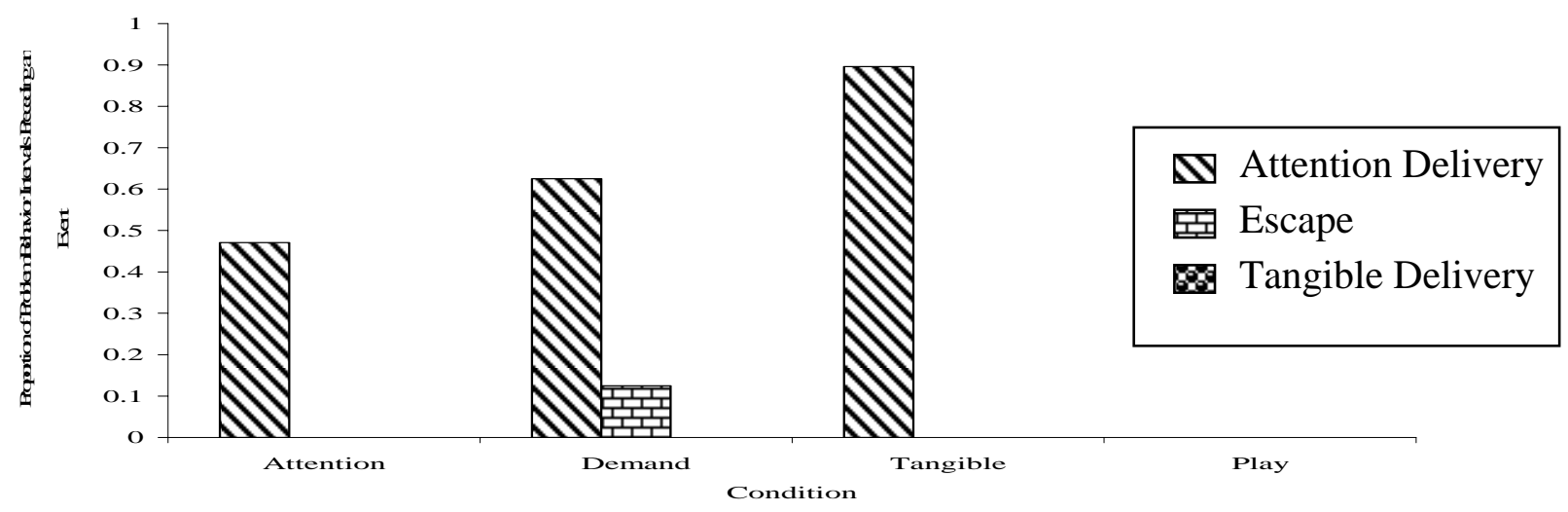


Figure 4
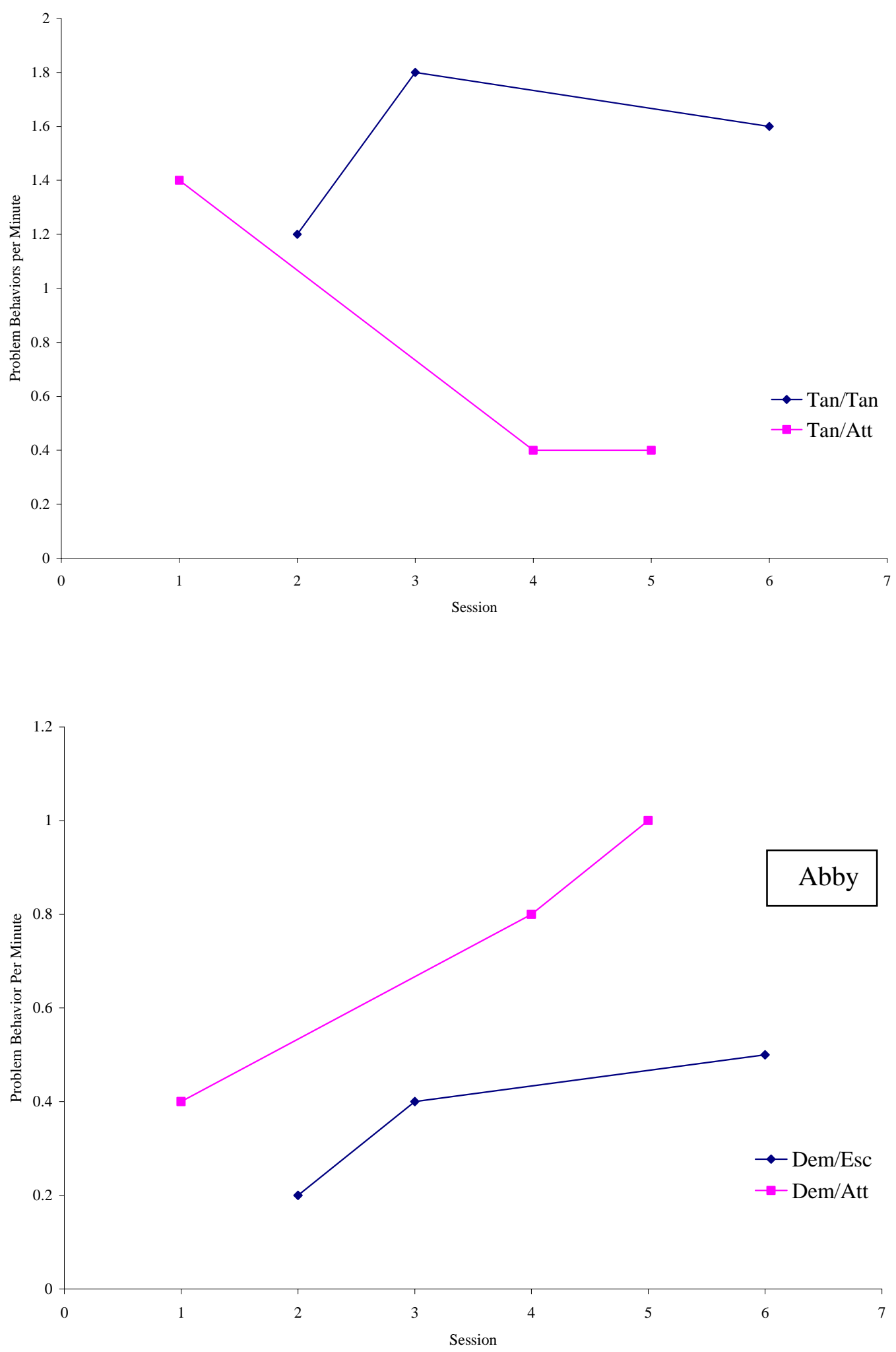